\title{
Ersetzen Ärztinnen und Ärzte bald die Computer?
}

\section{Daniel Strassberg}

Facharzt für Psychiatrie und Psychotherapie, Philosoph, Mitglied FMH

Es war Iwan Iljitsch klar, dass er sterben müsse, und darum befand er sich im Zustand ständiger Verzweiflung.

In seinem tiefsten Innern wusste Iwan Iljitsch, dass er sterben müsse, allein er wollte sich nicht nur nicht an diesen Gedanken gewöhnen, sondern konnte ihn einfach nicht begreifen, die nackte Tatsache nicht begreifen.

Jenes bekannte Beispiel für Syllogismen, das er in der Logik von Kiesewetter gelernt hatte: Cajus ist ein Mensch, alle Menschen sind sterblich, also ist auch Cajus sterblich, war ihm sein ganzes Leben hindurch rechtmässigerweise lediglich als auf Cajus anwendbar vorgekommen, keinesfalls aber auf ihn, Iwan Iljitsch, selber. Jenes war der Mensch Cajus, der Mensch überhaupt, und für diesen war das Gesetz völlig gerechtfertigt; er indes war nicht Cajus und ebenso wenig der Mensch an sich, sondern er war ein Wesen völlig für sich und völlig von allen anderen verschieden; er war der Wanja mit seiner Mama und seinem Papa, mit Mitja und Wolodja, mit Spielzeug und einem Kutscher, mit seiner Kinderfrau und späterhin mit Katjenka, kurz, mit allen Freuden, Leiden und Entzückungen der Kinderzeit und Jugend. War denn der Geruch des aus Lederstreifen zusammengesetzten Balles, den Wanja so geliebt hatte, etwa für Cajus bestimmt gewesen?, und hatte Cajus etwa, so wie er, die Hand der Mama geküsst?, und hatte vielleicht für jenen die Seide des Faltenkleides der Mama gerauscht? War es etwa Cajus gewesen, der in der Rechtsschule wegen der Kuchen revoltiert hatte? Cajus, der so verliebt gewesen war wie er?, und verstand etwa Cajus so wie er eine Verhandlung zu leiten?

Cajus, der war in der Tat sterblich, und wenn er starb, so war es ganz in der Ordnung; ich dagegen, ich, Wanja, ich, Iwan Iljitsch, mit all meinen Gefühlen und Gedanken bei mir ist es nun einmal eine ganz andere Sache. Und es kann ja gar nicht sein, dass auch ich sterben muss. Das wäre zu entsetzlich.

Derart waren seine Gefühle. [1]

Präziser und eindringlicher kann auch keine teure wissenschaftliche Studie den Zwiespalt der heutigen Medizin darstellen. Man braucht bloss Syllogismus durch Algorithmus zu ersetzen.

Iwan Iljitsch besteht darauf, kein Exemplar der Gattung Mensch zu sein, der mit einem Syllogismus er- mittelt werden kann. Er will eine eigene unverwechselbare Person, mit einem Namen und einer Geschichte sein. Ein singulärer Mensch. Hätte er das algorithmische Denken schon gekannt, hätte er sich wohl auch dagegen gewehrt. Er will nicht das Ergebnis einer Kette von Berechnungen sein. Die Ärztin hingegen würde in ihm genau das sehen. Ja, sie müsste einen zeitgenössischen Iwan Iljitisch unter diesem Blickwinkel betrachten, wenn sie ihm eine optimale Behandlung zukommen lassen will.

Als Alan Turing 1950 sein imitation game vorstellte, ging es ihm um die Frage, ob Computer eines Tages wie

\section{Iwan Iljitsch besteht darauf, kein Exemplar} der Gattung Mensch zu sein, der mit einem Syllogismus ermittelt werden kann.

Menschen denken können. Die Anlage des Spiels, das als Turing-Test Weltberühmtheit erlangen sollte, war recht einfach: Eine oder mehrere Personen richten Fragen an eine andere Person beziehungsweise an einen Computer, die hinter einem Vorhang verborgen sind. Der Fragende weiss deshalb nicht, ob ihm ein Mensch oder ein Computer antwortet. Wenn, so Turings Annahme, der Fragende nach einer bestimmten Anzahl von Antworten nicht entscheiden kann, hinter welchem Vorhang der Mensch sitzt, dann besitzt der Computer künstliche Intelligenz. In der Literatur über diesen Test, wie über die künstliche Intelligenz überhaupt,

\section{CAS-Lehrgang Philosophie}

Der Arzt und Philosoph Daniel Strassberg gründete 2004 in Zürich den CAS-Lehrgang in Philosophie für Fachleute aus Medizin und Psychotherapie, den er auch leitet. Im nächsten Semester werden die Widersprüche der Aufklärung behandelt, in dem es unter anderem auch um die Folgen der Digitalisierung geht.

Beginn: 25. Oktober. Es sind noch Plätze frei.

Information und Anmeldung unter www.entresol.ch oder info[at]entresol.ch 
fehlt allerdings, was Turing völlig klar war: Man kann das Spiel auch umkehren. Der Mensch könnte lernen, einen Computer so gut zu imitieren, dass der Tester aus diesem Grund die Unterscheidung nicht mehr treffen kann. Dass sich aber menschliches Denken allmählich dem Computer anpassen könnte, ist noch beunruhigender, als dass der Computer menschliche Intelligenz besitzen könnte. [2] Tatsächlich scheint sich im Windschatten der aufgeregten öffentlichen Diskussion, ob Maschinen wie Menschen denken können, die umgekehrte Tendenz durchzusetzen: Menschen denken wie Computer. Vor allem Ärztinnen und Ärzte werden systematisch in algorithmischem Denken geschult, sie lernen zu denken wie ein Computer.

Ein Algorithmus ist nichts anderes als eine Transformationsregel, eine Anweisung also, wie ich von Ausgangszustand A mittels der Regel B zum Zustand C komme: Wenn Oberbauchbeschwerden, dann Gastroskopie, wenn Entzündung, dann Helicobacter testen, wenn Helicobacter positiv, dann Antibiotika. Es wird ein festgeschriebenes Programm abgeschnurrt. Wie bei einem Computer.

\section{Denken nach Transformationsregeln}

Wahrscheinlich ist dies nur in den wenigsten Fällen ein Nachteil. In den allermeisten Fällen mag algorithmisches Wenn-dann-Denken gegenüber dem Erfassen von Gestalten Vorteile haben: Es funktioniert unabhängig von persönlicher Erfahrung, es kann auch vom Anfänger mit entsprechendem Handbuch korrekt ausgeführt werden, es garantiert eine unité de doctrine, es kann sauber beforscht werden, und es vermeidet blinde Flecken durch idiosynkratische Neigungen. Wahrscheinlich ist es auch billiger, weil immer dieselben Routinen wiederholt werden können. Vor allem aber sichert es gegen Klagen ab.

Abgesehen von der Tatsache, dass Algorithmen den Arztberuf über kurz oder lang überflüssig machen, weil Computer diese Arbeiten bald schneller und genauer erledigen werden, hat diese Art zu denken einen gewaltigen Nachteil: Selbstverhältnisse sind nicht algorithmisch.

Der moderne Iwan Iljitsch, der den Arzt wegen eines Prostatakarzinoms aufsucht, will sich weder als Resultat einer Wahrscheinlichkeitsrechnung noch als Exemplar einer Gattung verstehen. Er sieht sich als absolut singuläre Gestalt. Um ihn aber optimal zu behandeln, muss ihn die Ärztin als Ergebnis eines Kalküls begreifen, sie muss Algorithmen über ihn laufen lassen, ihn sozusagen aus einer möglichst grossen Datenbasis her- ausrechnen. Sie füttert den Computer mit Alter, histologischen Typus und Ausbreitung des Karzinoms, und Algorithmen errechnen daraus die optimale Therapie für diesen besonderen Fall. Je mehr Daten zur Verfügung stehen, desto näher kann man auf den Einzelfall einzoomen, im Idealfall sogar, so die Hoffnung, auf genau diesen einzelnen Menschen. Dies nennt sich dann personalisierte Medizin.

Doch die personalisierte Medizin ist ein Etikettenschwindel. Selbst bei einem extrem feinen Raster und unendlich vielen Verzweigungen der Algorithmen resultiert nicht eine singuläre Person, sondern eine Gruppe mit spezifischen Eigenschaften. Bestenfalls eine Gruppe mit $\mathrm{n}=1$.

Iwan Iljitsch versteht sich selbst nämlich nicht deshalb als Singularität, als eine einmalige Person, weil er keine Eigenschaften mit einer anderen Person teilt, sondern weil er eine unverwechselbare Geschichte hat. Menschen bilden ihre Identität nicht aufgrund einer Liste Eigenschaften aus - das interessiert nur das Passbüro -, sondern aufgrund von Erzählungen über ihr Leben und ihre Beziehungen.

\section{Die Kluft zwischen Arzt und Patient}

Genau deswegen verstärkt algorithmisches Denken die Kluft zwischen Arzt und Patient, bis zum Punkt, wo sie völlig aneinander vorbeireden. Das Bild, das der Patient von sich selbst hat, und das Bild, das der Arzt von ihm hat, können nicht miteinander zur Deckung gebracht werden, und zwar weil sie sich aus völlig unterschiedlichen Elementen zusammensetzen. Das eine bezieht sich auf Daten, das andere auf Geschichten. Wenn in naher Zukunft die Algorithmen die traditionellen Aufgaben der Ärztin, des Arztes übernehmen werden, bleibt diesem aber dennoch eine entscheidende Aufgabe: die des Übersetzers, der zwischen den unterschiedlichen Welten vermittelt. Doch die Kluft zwischen Daten und Geschichten kann weder durch einige Feigenblattkurse in medizinischer Ethik oder in Gesprächsführung überbrückt werden, noch durch wohlmeinende Aufrufe zur Empathie. Das Problem ist weder die Moral noch die fehlende Empathie, sondern das fehlende Wissen darüber, wie sich menschliche Identität konstituiert.

Literatur

1 Leo Tolstoi, Der Tod des Iwan Iljitsch, Stuttgart 1992, S. 55-6.

2 Alan M. Turing, «Kann eine Maschine denken?», in: Walter Ch. Zimmerli / Stefan Wolf, Künstliche Intelligenz. Philosophische Probleme, Stuttgart 1994, S. 39-78. 